\title{
State of the Art and Uses for the Biopharmaceutics Drug Disposition Classification System (BDDCS): New Additions, Revisions, and Citation References
}

\author{
Giovanni Bocci, ${ }^{1,2,3}$ Tudor I. Oprea,,$^{2,4,5,6,7}$ and Leslie Z. Benet ${ }^{1,8}(D)$
}

Received 10 November 2021; accepted 24 January 2022; published online 23 February 2022

\begin{abstract}
The Biopharmaceutics Drug Disposition Classification system (BDDCS) is a four-class approach based on water solubility and extent of metabolism/permeability rate. Based on the BDDCS class to which a drug is assigned, it is possible to predict the role of metabolic enzymes and transporters on the drug disposition of a new molecular entity (NME) prior to its administration to animals or humans. Here, we report a total of 1475 drugs and active metabolites to which the BDDCS is applied. Of these, 379 are new entries, and 1096 are revisions of former classification studies with the addition of references for the approved maximum dose strength, extent of the systemically available drug excreted unchanged in the urine, and lowest solubility over the $\mathrm{pH}$ range 1.0-6.8 when such information is available in the literature. We detail revised class assignments of previously misclassified drugs and the literature analyses to classify new drugs. We review the process of solubility assessment for NMEs prior to drug dosing in humans and approved dose classification, as well as the comparison of Biopharmaceutics Classification System (BCS) versus BDDCS assignment. We detail the uses of BDDCS in predicting, prior to dosing animals or humans, disposition characteristics, potential brain penetration, food effect, and drug-induced liver injury (DILI) potential. This work provides an update on the current status of the BDDCS and its uses in the drug development process.
\end{abstract}

KEY WORDS: BDDCS; BCS; DILI; dose number; extent of metabolism; food effects; solubility.

\section{INTRODUCTION}

The Biopharmaceutics Drug Disposition Classification System (BDDCS), based on rate of membrane permeability/ extent of metabolism and solubility characteristics, was proposed by $\mathrm{Wu}$ and Benet (1) as a methodology to predict drug disposition properties. This manuscript reports the

\footnotetext{
${ }^{1}$ Department of Bioengineering and Therapeutic Sciences, Schools of Pharmacy and Medicine, University of California, San Francisco, California, 94143-0912, United States of America.

${ }^{2}$ Translational Informatics Division, Department of Internal Medicine, University of New Mexico, Albuquerque, New Mexico 87131, United States of America.

${ }^{3}$ ExScientia, The Schrödinger Building, Oxford Science Park, Oxford, OX4 4GE, UK.

${ }^{4}$ UNM Comprehensive Cancer Center, Albuquerque, New Mexico 87131, United States of America.

${ }^{5}$ Department of Rheumatology and Inflammation Research, Institute of Medicine, Sahlgrenska Academy at Gothenburg University, Gothenburg, Sweden.

${ }^{6}$ Novo Nordisk Foundation Center for Protein Research, Faculty of Health and Medical Sciences, University of Copenhagen, Copenhagen, Denmark.

${ }^{7}$ Roivant Discovery, 451 D Street, Boston, MA 02210, USA.
}

BDDCS class for many newly approved drugs and revisits previously published BDDCS collection articles, providing citation references for reported parameters and, in some cases, correcting the classifications previously reported. We update the solubility criterion that has been proposed for the early classification of drugs prior to determination of the human dose; compare Biopharmaceutics Classification

\footnotetext{
${ }^{8}$ To whom correspondence should be addressed. (e-mail: leslie.benet@ucsf.edu)

Abbreviations: $B C S$, Biopharmaceutics Classification System; BDDCS, Biopharmaceutics Drug Disposition Classification System; $D I L I$, Drug-induced liver injury; $D N$, Dose number; ECCS, Extended clearance classification system; EoM, Extent of metabolism; $F_{a b s}$, Fraction of an oral dose that is absorbed through the wall of the gastrointestinal tract; $f_{e}$, Fraction of the systemically available dose that is excreted unchanged in urine; $F_{\text {extent }}$, Fraction of an oral dose that reaches the systemic circulation; $F_{g}$, Fraction of the absorbed oral dose that is not metabolized in the intestine; $M D S$, Approved maximum dose strength; $N M E$, New molecular entity; $O A T$, Organic anion transporter; $O A T P$, Organic anion transporter polypeptide; $P A M P A$, Parallel artificial membrane permeability assay; $P B P K$, Physiologically based pharmacokinetic; P-gp, P-glycoprotein; $S O L_{F D A}$, The lowest drug solubility measured across the $\mathrm{pH}$ range $1-6.8 ; T_{\text {peak }}$, Time of peak concentration following an oral dose.
} 
System (BCS) assignments with BDDCS assignments when the former are available; and review uses/insights that BDDCS classification provides in early drug development before a new molecular entity (NME) is dosed to animals or humans.

\section{THE DIFFERENCES BETWEEN BCS AND BDDCS ASSIGNMENT}

The BCS, proposed by Amidon et al. (2), was developed to reduce the burden of conducting in vivo human studies related to regulatory approval and development of new formulations of immediate-release products. Drugs are classified in BCS based on the extent of permeability and the solubility of the active species present in an approved drug product (3). Drugs for which the extent of absorption is greater than $85 \%$ (high extent of permeability) are designated as BCS class 1 or 2, while drugs not achieving a high extent of permeability are designated as BCS classes 3 and 4. Further separation is based on measured solubility depending on the dose number (DN). In BDDCS, this parameter is calculated based on the previous FDA criteria of the approved maximum dose strength (MDS), which is the highest approved dose of the drug in milligrams, the drug water solubility as defined by the FDA criterion ( $\mathrm{SOL}_{\mathrm{FDA}}$ ), which is the lowest drug water solubility $(\mathrm{mg} / \mathrm{mL})$ measured across the $\mathrm{pH}$ range 1-6.8 and the assumed human gastric volume of $250 \mathrm{~mL}$. These three parameters are necessary to calculate the $\mathrm{DN}\left(\mathrm{DN}=\frac{M D S}{S O L_{F D A} \cdot 250}\right)$. BDDCS classification utilizes the same DN characteristics as BCS for approved drugs prior to May 2021 to differentiate classes 1 and 3 (high solubility) from classes 2 and 4 (low solubility). The updated BCS regulations harmonized through $\mathrm{ICH}$ (3) now defines solubility in terms of the highest single therapeutic dose. The effect of this difference will be discussed in a subsequent section.

However, Wu and Benet (1) recognized that the rate of intestinal permeability (rather than the extent) could lead to the prediction of the extent of metabolism (EoM) of a drug. The high intestinal permeability rate is the defining characteristic of BDDCS classes 1 and 2 drugs, while low intestinal permeability rate is the defining characteristic for BDDCS classes 3 and 4 drugs. Passive drug membrane permeability rate in any relevant membrane such as a Caco-2 cell line or even a nonbiologic PAMPA (4) provides a reasonable estimate of EoM. Wu and Benet (1) reported that the vast majority of approved drugs were either EoM $\geq 70 \%$ or EoM $\leq 30 \%$, easily separating BDDCS classes 1 and 2 drugs from classes 3 and 4 drugs. The fraction of the available dose that is excreted unchanged in urine $\left(f_{e}\right)$ can be translated into a measurement of a drug's EoM. Drugs exhibiting $f_{e}$ values $\leq$ $30 \%$ were considered extensively metabolized, high permeability BDDCS classes 1 and 2 . This estimate could be confounded by marked biliary elimination of unchanged drug, but information concerning a drug's metabolic elimination and potential biliary elimination was considered in making the BDDCS assignment.

$\mathrm{Wu}$ and Benet (1) further reasoned that poor passive permeability drugs (BDDCS classes 3 and 4) would require transporters to achieve membrane permeability, but that transporters may not significantly affect drug disposition for high permeability rate drugs, especially for highly soluble BDDCS class 1 drugs where high concentrations of drug would be available for passive diffusion. Therefore, although the high permeability rate BDDCS class 2 drugs are primarily metabolized, transporters may or may not be clinically relevant in drug disposition due to the lower available concentration resulting from their low solubility characteristics.

\section{NEW ADDITIONS TO BDDCS}

Although the BDDCS was first introduced in 2005 (1), the two major works listing drugs and their BDDCS class are the 2011 paper of Benet et al.(5) and the 2016 paper by Hosey et al.(6). Since then, no further multiple BDDCS classifications were provided to the scientific community. Our work here aims to provide new BDDCS assignments for drugs not previously listed. We compiled a list of 140 drugs approved between 2017 and 2020 enriched with older drugs that were not previously classified for a final number of 379 newly classified drugs. We carefully inspected the literature to retrieve the information necessary for assessing the BDDCS class of these 379 additional drugs and reviewed the previously listed 1096 assignments. The results of these new assignments are depicted in Fig. 1, together with the previous classification of Benet et al. (5) and Hosey et al. (6) and the distribution of the total 1475 compounds. For a few drugs, the value of $f_{e}$ can be susceptible to urine $\mathrm{pH}$ changes, so much so that classification can change from classes 1 and 3 to classes 2 and 4 depending on urine $\mathrm{pH}$. These drugs are listed as BDDCS class 0 . For the previously 379 BDDCS unclassified drugs, we report 151 class 1,147 class 2, 52 class 3, and 29 class 4 drugs. The list of these newly classified BDDCS drugs can be found in Table I and in Supporting information. The distribution of BDDCS class for newly approved drugs since 2017 in our analysis is presented in Fig. 2, demonstrating the predominance of class 2 , followed by class 1 .

For the drugs lusutrombopag and binimetinib, a precise class could not be assigned. These two drugs are extensively metabolized, but we could not find any information regarding their solubility. However, no food effects are reported in their labels, which suggest that their classification is BDDCS 1 drugs $(1,7)$.

\section{BDDCS REVISIONS}

Hosey and co-workers (6) identified some drugs that had been previously misclassified either because their EoM was wrongly annotated or because biliary excretion was not considered, when it was the predominant elimination route for the unchanged drug. Upon applying these corrections, drugs were correctly reclassified to different BDDCS classes (6). Here, we extend the revision work to the 1096 drugs reported previously $(1,5)$ by reviewing EoM and SOLFDA data reporting each value and the reference(s) with supporting data. We also made a number of BDDCS classification revisions. In Table II, we summarize the results of the BDDCS revision work.

The great majority of the reviewed drugs $(92.2 \%)$ retain their former BDDCS assigned class. Of the drugs that had a class change, the vast majority had a single property class 


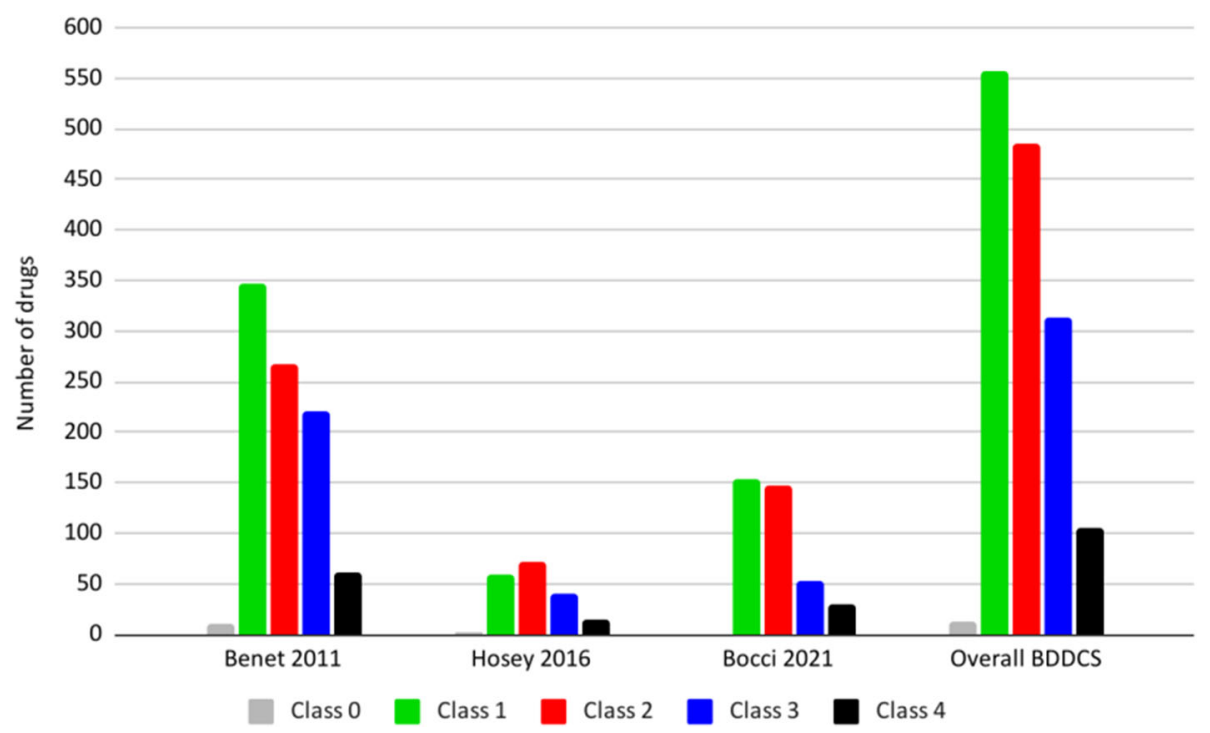

Fig. 1 State of the art for the drugs classified with the BDDCS across all collections over time

change, which means that either the EoM or the SOL updated value caused the change in class. For example, 18 BDDCS class 1 and five BDDCS class 3 drugs were found to have a low solubility in the literature. Since their EoM was confirmed, these drugs were reassigned to either BDDCS class 2 or 4, respectively. Alternatively, the solubilities of 24 BDDCS class 2 and 22 BDDCS class 4 drugs were, instead, found to be high, while their EoMs were substantially confirmed. Thus, 24 previously listed BDDCS class 2 drugs were reassigned as class 1 , and 22 class 4 drugs were reassigned as class 3. Furthermore, the EoM values for seven BDDCS class 1 and four BDDCS class 2 drugs were found to be low in the literature, with no critical changes in solubility. Hence, these drugs were reassigned as BDDCS class 3 and class 4, respectively. Moreover, where solubility values were confirmed, there were a few cases where the opposite class change occurred. Similarly, three BDDCS class 3 drugs were re-classified as BDDCS class 1 because their EoM were high. However, we do not report any class change from BDDCS class 4 to BDDCS class 2. The only drug for which we detected a double property class change is fialuridine, which is revised from BDDCS class 2 to BDDCS class 3. The complete list of 84 drugs for which we report a change of BDDCS class is in Table III. Complete revision details are provided in Supporting information Table S3.

\section{DISCREPANCIES BETWEEN BDDCS AND BCS PREDICTIONS}

Major drug regulatory agencies use the BCS (2) to assess the eligibility of drugs for a waiver of in vivo bioequivalence studies $(3,8)$. In other words, two drug products containing the same drug substance can be considered bioequivalent if their rate and extent of availability (after oral administration, at the same molar dose) lie within acceptable predefined limits. BCS classes 1 (high solubility, high permeability) and 3 (high solubility, low permeability) immediate-release orally dosed drugs are eligible for biowaivers. The list of the 257 drugs for which we could determine both BCS and BDDCS classification is in Table IV. Almost all of these 257 drugs were assigned their BCS class based on the previous MDS solubility criterion, not the revised highest therapeutic dose criterion (3); therefore in this compilation, we continue to use MDS in the BDDCS classification. We believe this change in BCS criteria will have little if any impact on the usefulness of BDDCS, Because of confidentiality issues, regulatory agencies do not identify the number or the names of specific drugs eligible for a biowaiver, and we have no way of knowing whether these published BCS classifications have been allowed biowaivers, yet since we could only locate a BCS class designation for 257 of the 1475 BDDCS classified drugs detailed here, we believe that regulatory agencies have accepted relatively few drugs to be biowaiver eligible. BCS classes 2 (low solubility, high permeability) and 4 (low solubility, low permeability) drugs are not eligible. The BDDCS was intended to expand the number of BCS classes 1 and 3 drugs eligible for a biowaiver (for drugs not BCS classified) and predict all drugs' disposition profiles (3). However, as noted by Metry and Polli (9), the harmonized BCS criteria will lead to even fewer drugs eligible for biowaivers.

BCS class assignment is ambiguous in some cases because the permeability assignment relies on absorption measurements in humans that are often uncertain and difficult to perform and the lack of intravenous dosing data. Supporting information Table S5 lists the BCS classification for all drugs with appropriate references. Table $\mathrm{V}$ summarizes the agreement between the two classification systems. Classification differences between BCS and BDDCS are caused by two factors. First is the definition of permeability. In BCS, high permeability refers to high extent of absorption (greater than $85 \%$ ) whereas in BDDCS high permeability refers to a high rate of permeability. Therefore, it is possible that a BCS class 1 drug would be classified as BDDCS class 3 if it has a low permeability rate, but the overall extent of absorption is high. The 13 BCS class 1 drugs in Tables IV and V that are BDDCS class 3 are probably due to this reason. For biowaivers, this difference is not relevant since both BCS classes 1 and 3 drugs are eligible. However, predictions of the importance olf transporters in the disposition of these drugs 
Table I New BDDCS classifications

\section{Name}

Abametapir

Abemaciclib

Abiraterone

Abiraterone acetate

Acalabrutinib

Acemetacin

Acenocoumarol

Acetylcholine chloride

Acetylmethadol

Adefovir

Ademetionine butane disulfonate

Adinazolam

Afamelanotide acetate

Alatrofloxacin mesylate

Alectinib hydrochloride

Alimemazine tartrate

Alizapride hydrochloride

Alogliptin

Alpelisib

Ambenonium chloride

Amifampridine phosphate

Aminolevulinic acid hydrochloride

Amobarbital sodium

Amodiaquine

Anagrelide hydrochloride

Antazoline mesylate

Antofloxacin hydrochloride

Apalutamide

Apixaban

Arbekacin

Arbutin

Arsenic trioxide

Artemisinin

Artesunate

Atrasentan hydrochloride

Aurothioglucose hydrate

Avapritinib

Avatrombopag maleate

Avibactam

Azatadine

Bacampicillin hydrochloride

Baloxavir

Baloxavir marboxil

Balsalazide disodium

Baricitinib

Beclomethasone dipropionate

Bempedoic acid

Benfluorex hydrochloride

Benzthiazide

Berotralstat hydrochloride

Betahistine dihydrochloride

Betrixaban maleate

Bictegravir sodium

Binimetinib

Bisacodyl

Bleomycin sulfate

Brexanolone

Brigatinib

Brivaracetam

Brivudine

Bromopride
Table I. (continued)

Name

BDDCS

Brompheniramine maleate

Bunazosin

Buserelin acetate

Butobarbital sodium

Cabazitaxel

Calcitonin (salmon synthetic)

Camylofine dihydrochloride

Cangrelor tetrasodium

Cannabidiol

Capmatinib hydrochloride

Carboprost tromethamine

Carglumic acid

Cedazuridine

Cefcanel daloxate hydrochloride

Cefetamet

Cefetamet pivoxil

Cefiderocol sulfate tosylate

Cefozopran hydrochloride

Cenobamate

Cholestyramine

Cibenzoline

Clascoterone

Clemizole hydrochloride

Clenbuterol hydrochloride

Clobetasol propionate

Cobimetinib fumarate

Colestipol

Copanlisib dihydrochloride

Crisaborole

Cyclothiazide

Dacomitinib

Dapoxetine hydrochloride

Darolutamide

Decitabine

Deferoxamine mesylate

Deflazacort

Delafloxacin meglumine

Deutetrabenazine

Dexbrompheniramine

Dexchlorpheniramine

Dexlansoprazole

Dexmedetomidine hydrochloride

Dichloroacetic acid

Dichlorphenamide

Dicyclomine hydrochloride

Diethylpropion hydrochloride

Dihydrocodeine bitartrate

Dihydrodydrogesterone

Diphenoxylate hydrochloride

Dirithromycin

Doravirine

Doxacurium chloride

Doxapram hydrochloride

Doxylamine succinate

Droperidol

Drotaverine

Droxidopa

Duvelisib

Dydrogesterone

Dyphylline

Econazole nitrate

1

1

2

2

1

1

1 1

1

2

4

1

3

2 
Table I. (continued)

\begin{tabular}{ll}
\hline Name & BDDCS
\end{tabular}

Edaravone

Elagolix sodium

Elbasvir

Elexacaftor

Enasidenib mesylate

Encorafenib

Enoximone

Entrectinib

Ephedrine

Epinastine hydrochloride

Epinephrine

Eravacycline dihydrochloride

Erdafitinib

Eribulin mesylate

Ertugliflozin L-pyroglutamic acid

Eslicarbazepine

Estramustine

Estramustine phosphate

Estriol

Etelcalcetide hydrochloride

Ethacrynic acid

Ethionamide

Ethoxzolamide

Ethylene glycol

Ethynodiol diacetate

Etofibrate

Etretinate

Favipiravir

Fedratinib dihydrochloride

Fenoldopam mesylate

Ferric maltol

Floxuridine

Fluorescein sodium

Fominoben

Fondaparinux

Fosnetupitant chloride hydrochloride

Fosphenytoin sodium

Fospropofol disodium

Fostamatinib disodium hexahydrate

Fostemsavir tromethamine

Furamidine

Furazolidone

Gabapentin enacarbil

Gabexate mesylate

Gadofosveset trisodium

Gadoteridol

Gamma hydroxybutyric acid

Garenoxacin mesylate

Gatifloxacin

Gemifloxacin mesylate

Gilteritinib fumarate

Givosiran

Glasdegib maleate

Glecaprevir

Glucose

Glutethimide

Glycerol

Glycerol phenylbutyrate

Grazoprevir

Guaifenesin

Halofantrine
Table I. (continued)

\begin{tabular}{ll}
\hline Name & BDDCS
\end{tabular}

Ibrexafungerp 2

Infigratinib 2

Irofulven 2

Isomazole $\quad 1$

Isoxicam $\quad 2$

Istradefylline $\quad 2$

Ivosidenib 2

Ixazomib 1

Ketobemidone 1

Lactitol 1

Lactose 1

Lactulose

Larotrectinib sulfate

Lasmiditan hemisuccinate

Lefamulin acetate

Lemborexant

Lercanidipine hydrochloride

Letermovir

Levocarnitine

Levoleucovorin

Levomethadyl acetate hydrochloride

Levorphanol tartrate $\quad 1$

Linagliptin 3

Lindane 2

Lomustine $\quad 2$

Lonafarnib 2

Lorlatinib 2

Lormetazepam $\quad 2$

Loxapine succinate 1

Lubiprostone 2

Lumateperone tosylate 1

Lurbinectedin 2

Lusutrombopag $\quad 1$

Macitentan $\quad 2$

Mannitol 3

Mazindol 1

Melagatran $\quad 4$

Melperone 1

Mepenzolate 1

Metazosin 4

Methacycline $\quad 3$

Methionine 1

Methsuximide 1

Methylparaben 1

Methyltestosterone $\quad 2$

Meticrane 4

Metildigoxin 3

Mevastatin $\quad 2$

Midodrine hydrochloride $\quad 1$

Midostaurin 2

Migalastat hydrochloride $\quad 3$

Mitiglinide 1

Mitomycin 1

Mitotane $\quad 2$

Mizoribine $\quad 3$

Moclobemide 1

Moexipril hydrochloride $\quad 1$

Moexiprilat 3

Moxidectin $\quad 2$

Moxonidine 4

Nabilone 2 
Table I. (continued)
Table I. (continued)

\begin{tabular}{|c|c|c|c|}
\hline Name & BDDCS & Name & BDDCS \\
\hline Naftopidil & 2 & Rifamycin sodium & 3 \\
\hline Naldemedine tosylate & 1 & Rifapentine & 2 \\
\hline Nandrolone & 1 & Rimegepant sulfate & 4 \\
\hline Nandrolone decanoate & 2 & Ripretinib & 2 \\
\hline Nebivolol hydrochloride & 2 & Risdiplam & 2 \\
\hline Neratinib maleate & 2 & Rivaroxaban & 2 \\
\hline Netarsudil dimesylate & 1 & Rucaparib camsylate & 2 \\
\hline Netupitant & 2 & Safinamide mesylate & 1 \\
\hline Niraparib tosylate & 1 & Samidorphan & 1 \\
\hline Nitazoxanide & 2 & Sarecycline hydrochloride & 3 \\
\hline Noradrenaline & 1 & Secnidazole & 1 \\
\hline Obeticholic acid & 2 & Selinexor & 2 \\
\hline Oliceridine fumarate & 1 & Selpercatinib & 1 \\
\hline Olsalazine sodium & 1 & Selumetinib sulfate & 1 \\
\hline Omadacycline tosylate & 3 & Semaglutide & 2 \\
\hline Opicapone & 2 & Sematilide hydrochloride & 3 \\
\hline Osilodrostat phosphate & 1 & Semaxanib & 2 \\
\hline Oxyphenbutazone & 2 & Setmelanotide acetate & 3 \\
\hline Oxyphenonium bromide & 1 & Sevelamer & 4 \\
\hline Oxytocin & 1 & Silodosin & 2 \\
\hline Ozanimod hydrochloride & 1 & Siponimod fumarate & 2 \\
\hline Pafuramidine & 2 & Sitaxentan sodium & 1 \\
\hline Papaverine hydrochloride & 1 & Solriamfetol hydrochloride & 3 \\
\hline Pegaptanib sodium & 1 & Sorbitol & 1 \\
\hline Pemigatinib & 2 & Sorivudine & 2 \\
\hline Pentachlorophenol & 2 & Sotorasib & 2 \\
\hline Perphenazine & 2 & Stanozolol & 1 \\
\hline Pexidartinib hydrochloride & 2 & Stiripentol & 1 \\
\hline Phenelzine sulfate & 1 & Succimer & 1 \\
\hline Pheniramine maleate & 1 & Sulfaphenazole & 2 \\
\hline Phenol & 1 & Tafamidis & 2 \\
\hline Phenprocoumon & 1 & Tafamidis meglumine & 2 \\
\hline Pibrentasvir & 4 & Tafenoquine succinate & 2 \\
\hline Pidotimod & 3 & Talazoparib tosylate & 3 \\
\hline Pilsicainide hydrochloride & 3 & Tapentadol hydrochloride & 1 \\
\hline Pimavanserin tartrate & 1 & Tazemetostat hydrobromide & 2 \\
\hline Pinaverium bromide & 1 & Tecovirimat & 2 \\
\hline Piperacetazine & 1 & Tedizolid & 2 \\
\hline Pipobroman & 1 & Telbivudine & 3 \\
\hline Pitolisant hydrochloride & 1 & Telotristat & 2 \\
\hline Plazomicin sulfate & 3 & Telotristat ethyl etiprate & 2 \\
\hline Plecanatide & 1 & Temsavir & 2 \\
\hline Polythiazide & 2 & Tenapanor hydrochloride & 2 \\
\hline Pralsetinib & 2 & Tenofovir & 3 \\
\hline Pranlukast & 2 & Tenofovir alafenamide fumarate & 1 \\
\hline Pregnenolone & 2 & Tezacaftor & 2 \\
\hline Pretomanid & 2 & Theobromine & 1 \\
\hline Pridinol & 2 & Tizoxanide & 4 \\
\hline Procarbazine hydrochloride & 1 & Tranilast & 2 \\
\hline Propiverine hydrochloride & 1 & Trichlormethiazide & 3 \\
\hline Propylparaben & 2 & Triclosan & 2 \\
\hline Prucalopride succinate & 3 & Trifarotene & 2 \\
\hline Rasagiline mesylate & 1 & Triheptanoin & 2 \\
\hline Recainam & 3 & Trimetaphan & 2 \\
\hline Relebactam & 3 & Trimethobenzamide hydrochloride & 2 \\
\hline Relugolix & 2 & Tripelennamine hydrochloride & 1 \\
\hline Remdesivir & 2 & Triprolidine hydrochloride & 1 \\
\hline Remimazolam besylate & 1 & Troleandomycin & 2 \\
\hline Remoxipride hydrochloride & 1 & Tucatinib & 2 \\
\hline Revefenacin & 1 & Ubrogepant & 2 \\
\hline Ribociclib succinate & 1 & Upadacitinib & 1 \\
\hline
\end{tabular}


Table I. (continued)

\begin{tabular}{ll}
\hline Name & BDDCS \\
\hline Uracil mustard & 1 \\
Vaborbactam & 4 \\
Valbenazine ditosylate & 2 \\
Valpromide & 2 \\
Velpatasvir & 4 \\
Venetoclax & 2 \\
Vibegron & 4 \\
Vildagliptin & 1 \\
Viloxazine hydrochloride & 1 \\
Voxelotor & 2 \\
Voxilaprevir & 4 \\
Ximelagatran & 2 \\
Zanubrutinib & 2 \\
Zimeldine & 1 \\
Zuclopenthixol dihydrochloride & 1 \\
\hline
\end{tabular}

are less accurate using the BCS class 1 designation. The second factor leading to differences in BCS and BDDCS assignment is the lesser accuracy of in vitro permeability measures in BCS translating to extent of permeability versus the accuracy of EoM assessments utilized in BDDCS. As Wu and Benet (1) state, the use of EoM over permeability (i.e., BDDCS over BCS) is preferable because after drug approval, it is easier to quantify EoM than extent of absorption as reflected in the multiple BCS assignments for many drugs as shown in Table IV. As expected, a large fraction of BCS classes 1, 2, and 3 are in agreement with their corresponding BDDCS classes $(69 \%, 81 \%$, and $64 \%$ respectively); thus, confirming the somewhat decent correlation between extent of absorption and extent of metabolism of drugs. However, the agreement drops markedly for BCS class 4 drugs, where only 4 out of $17(23 \%)$ are confirmed as BDDCS class 4 drugs. It is worth noting that the BCS class 4 drugs azathioprine, loperamide, meclizine, ribociclib, and selumetinib, utilizing the BDDCS classification based on the solubility values referenced here, would have made them eligible for a biowaiver. This discrepancy emerges from both their high extent of metabolism and from suspected errors in solubility class assignments. From our analysis, these drugs should be classified as BDDCS 1 . This difference in the permeability criteria makes it much simpler to assign BDDCS class versus BCS class. This observation is supported by the number of drugs currently classified by the two methods (not even 300 for BCS versus almost 1500 for BDDCS).

\section{ASSIGNMENT OF BDDCS CLASS FOR AN NME PRIOR TO IN VIVO STUDIES}

BCS class assignment can only be made after MDS is established allowing DN to be determined. This is not a limitation since the objective of BCS is to reduce the burden of conducting in vivo human studies related to regulatory approval of new formulations of immediate-release products. As presented above, this limitation is also true for BDDCS since DN and the extent of metabolism in humans are required. However, since the primary purpose of BDDCS is to predict drug disposition characteristics, it would be very useful if the BDDCS criteria could be adapted to allow classification of an NME before in vivo studies in animals and humans. The observed excellent correlation between the rate of membrane permeability and the extent of metabolism, first recognized by $\mathrm{Wu}$ and Benet (1), allows measures of in vitro membrane permeability to differentiate BDDCS classes 1 and 2 drugs from BDDCS classes 3 and 4 drugs prior to in vivo studies (4). However, as noted above, membrane permeability measurements can be variable, and therefore, the methodology with appropriate standards must be developed in each laboratory carrying out such analyses.

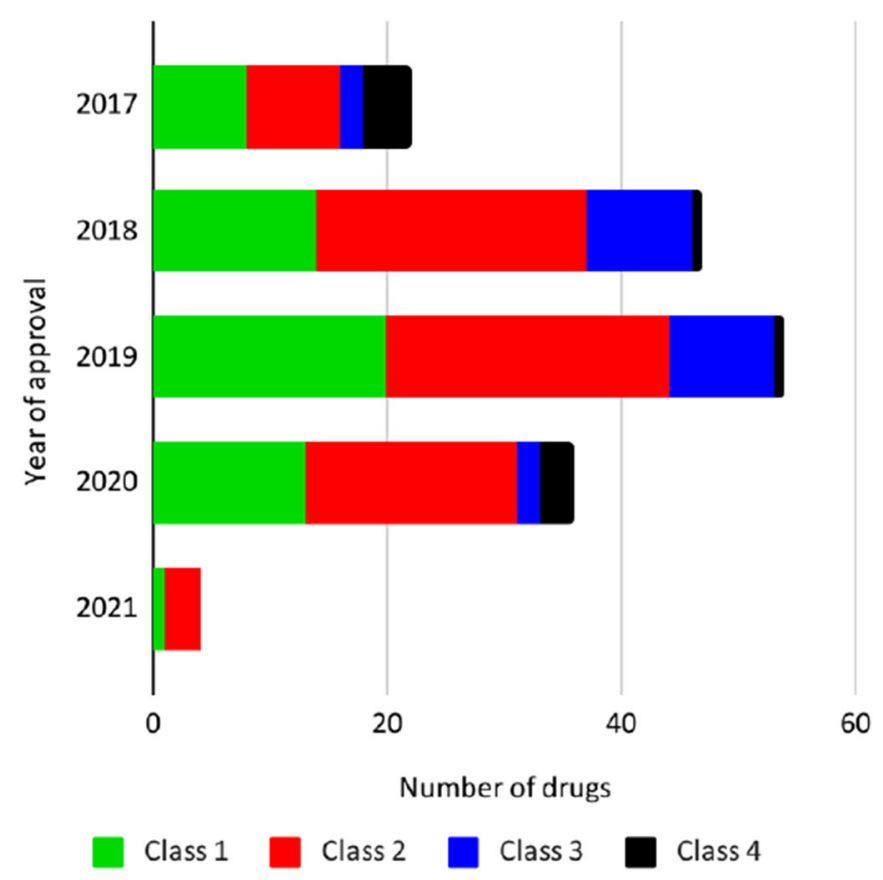

Fig. 2 BDDCS classes distribution over recent years 
Table II BDDCS class changes from former publications. The number of drugs with: BDDCS class unchanged (yellow), single property BDDCS class change (orange), double property BDDCS class change (red)

\begin{tabular}{|c|c|c|c|c|}
\hline & Updated Class 1 & Updated Class 2 & Updated Class 3 & Updated Class 4 \\
\hline Prior Listed as Class 1 & 378 & 18 & 7 & \\
\hline Prior Listed as Class 2 & 24 & 314 & 1 & 4 \\
\hline Prior Listed as Class 3 & 3 & & 252 & 5 \\
\hline Prior Listed as Class 4 & & & 22 & 54 \\
\hline
\end{tabular}

\section{The Solubility Classification Rule}

In 2016, Dave and Morris attempted to define an "early development classification rule" for solubility that could be applied in earlier phases of NME development (10). They reported that by applying a cutoff at $0.3 \mathrm{mg} / \mathrm{mL}$, it was possible to correctly assign BDDCS (and/or BCS) classes to $85 \%$ of the drugs for which a solubility value was reported by $\mathrm{Wu}$ and Benet $(1)$ at that time ( 600 drugs $)$. Hence, if the solubility of the NME is above $0.3 \mathrm{mg} / \mathrm{mL}$, it could be assigned to class 1 or 3 , whereas if its solubility is below or equal to $0.3 \mathrm{mg} / \mathrm{mL}$, the NME could be assigned to class 2 or 4. Since we have both updated and added new solubility values to the collection, we assessed if the $0.3 \mathrm{mg} / \mathrm{mL}$ cutoff is still optimal. Thus, we repeated the analysis done by Dave and Morris and screened cutoffs ranging from 0.1 to $1 \mathrm{mg} / \mathrm{mL}$ (i.e., including the 0.3 cutoff) with an increment of 0.01 . Not surprisingly, our results show that the 0.3 cutoff retains a remarkable accuracy of $87 \%$ (data not shown).

However, we identified a cutoff at $0.44 \mathrm{mg} / \mathrm{mL}$ that slightly increases the accuracy to $89 \%$ based on our cited solubility data for 1156 drugs. Table VI summarizes the number of correctly and incorrectly classified drugs if the 0.44 $\mathrm{mg} / \mathrm{mL}$ cutoff had been used before determining the dose number. Correct predictions would have been made for $87.9 \%$ of high solubility class $0 / 1 / 3$ drugs and $91.4 \%$ of poor solubility class $2 / 4$ drugs. It is worth noting that all 37 of class 2 or 4 drugs that were incorrectly predicted to be highly soluble are dosed at high quantities (MSD $\geq 150 \mathrm{mg}$ ), whereas 64 of 88 of class 1 or 3 drugs that were incorrectly predicted to be poorly soluble are dosed at low quantities $(\mathrm{MSD} \leq 10 \mathrm{mg})$.

Therefore, by using a measure of membrane permeability to differentiate classes 1 and 2 from classes 3 and 4 and using the $0.44 \mathrm{mg} / \mathrm{mL}$ solubility cutoff to differentiate classes 1 and 3 from classes 2 and 4, it is possible to assign a BDDCS classification to an NME before ever dosing the drug to animals or humans. We estimate that the correct prediction could be obtained for about $85 \%$ of small molecule NMEs. We came to this estimate based on the observation of $\mathrm{Wu}$ and Benet (1) that most of approved drugs were either EoM $\geq$ $70 \%$ or $\mathrm{EoM} \leq 30 \%$, combined with the above analysis that the $0.44 \mathrm{mg} / \mathrm{mL}$ cutoff provides accurate solubility prediction for about $90 \%$ of approved drugs. This allows drug development scientists to make reasonable predictions concerning the disposition of an NME early in drug development, as detailed below.

\section{POTENTIAL USES OF BDDCS ASSIGNMENT IN DRUG DEVELOPMENT}

\author{
Disposition of Drugs Based on BDDCS Assignment and \\ Potential Modulating Factors to be Considered in Disease \\ States, Drug-Drug Interactions, and Pharmacogenomic \\ Variance
}

As depicted in Fig. 3A, from $\mathrm{Wu}$ and Benet (1), the predominant route of elimination of BDDCS classes 1 and 2 drugs is via metabolism, both in the liver and intestine, while the predominant route of elimination of BDDCS classes 3 and 4 drugs is via excretion of unchanged drug in the urine or bile. As depicted in Fig. 3B, summarized by Shugarts and Benet (11), even when shown in vitro to be transporter substrates, most BDDCS class 1 drugs do not exhibit clinically significant transporter effects in the liver and intestine. In contrast, BDDCS classes 3 and 4 drugs are likely to exhibit clinically significant transporter effects in the liver and intestine because of their poor membrane permeability. BDDCS class 2 drugs, although predominantly eliminated by metabolism, can potentially exhibit both efflux and uptake transporter effects in the liver but only efflux transporter effects in the intestine.

Varma et al. (12) expanded the BDDCS findings to provide further predictions of liver and kidney clearance and gut bioavailability through their Extended Clearance Classification System (ECCS), which incorporated differentiation based on substrate molecular weight and charge status. For the ECCS listing of 363 drugs, in vitro permeability rate measured by the authors correctly predicted the major route of elimination for $89.5 \%$ of the drugs, confirming our conclusion above that in vitro permeability measurements provide good prediction of BDDCS classes 1 and 2 drugs versus classes 3 and 4 drugs as per Fig. 3A. The major predictions based on ECCS are (a) clearance of high molecular weight $(\geq 400 \mathrm{Da})$ acids and zwitterions (ECCS class $1 \mathrm{~B})$ will be rate-limited by hepatic organic anion transporter polypeptide (OATP) uptake; (b) more recently (13), it is hypothesized that clearance of low molecular weight ( $<400 \mathrm{Da})$ acids and zwitterions (ECCS class $1 \mathrm{~A})$ may be rate limited by organic anion transporter (OAT) uptake, although the clinical significance of this finding is not confirmed. As predicted by BDDCS the major route of elimination for high permeability ECCS class 1 compounds will be metabolism; (c) acids and zwitterions will not be appreciably metabolized by CYP3A, therefore $F_{g}$ (fraction of absorbed oral drug 
Table III BDDCS class changes from initial publications

\begin{tabular}{|c|c|c|c|}
\hline Name & Prior listed BDDCS & Corrected BDDCS & Initial publication \\
\hline Acarbose & 1 & 3 & (5) \\
\hline Adefovir dipivoxil & 3 & 1 & (5) \\
\hline Alpidem & 1 & 2 & (6) \\
\hline Amifloxacin & 3 & 4 & (6) \\
\hline Amineptine & 1 & 2 & (6) \\
\hline Artemether & 2 & 1 & (5) \\
\hline Azithromycin dihydrate & 3 & 4 & (5) \\
\hline Bendazac lysine & 2 & 1 & (6) \\
\hline Betamipron & 3 & 4 & (5) \\
\hline Bethanechol chloride & 3 & 1 & (6) \\
\hline Candesartan cilexetil & 4 & 2 & (5) \\
\hline Carbovir & 4 & 3 & (6) \\
\hline Cefadroxil & 3 & 4 & (5) \\
\hline Cefmetazole sodium & 3 & 4 & (5) \\
\hline Ceftazidime & 3 & 4 & (5) \\
\hline Chlorhexidine gluconate & 3 & 4 & (6) \\
\hline Cladribine & 2 & 1 & (5) \\
\hline Clinafloxacin & 3 & 4 & (6) \\
\hline Clodronic acid & 4 & 3 & (5) \\
\hline Daclatasvir dihydrochloride & 4 & 3 & (6) \\
\hline Daunorubicin & 2 & 1 & (5) \\
\hline Dexloxiglumide & 1 & 2 & (6) \\
\hline Dihydroergotamine mesylate & 1 & 2 & (6) \\
\hline Ergotamine tartrate & 1 & 2 & (5) \\
\hline Etoposide & 3 & 4 & (5) \\
\hline Everolimus & 1 & 2 & (5) \\
\hline Fialuridine & 2 & 3 & (6) \\
\hline Finasteride & 1 & 2 & (5) \\
\hline Fipexide & 3 & 2 & (6) \\
\hline Flavoxate hydrochloride & 2 & 1 & (6) \\
\hline Fluticasone propionate & 2 & 1 & (5) \\
\hline Fusidic acid sodium & 2 & 1 & (6) \\
\hline Genistein & 1 & 2 & (6) \\
\hline Guanethidine sulfate & 1 & 3 & (6) \\
\hline Lenalidomide & 4 & 3 & (5) \\
\hline Levonorgestrel & 4 & 2 & (5) \\
\hline Licarbazepine acetate & 1 & 2 & (6) \\
\hline Liothyronine sodium & 2 & 1 & (6) \\
\hline Loperamide hydrochloride & 3 & 1 & $(5)$ \\
\hline Medroxyprogesterone acetate & 4 & 2 & (5) \\
\hline Megestrol acetate & 4 & 2 & (5) \\
\hline Melphalan hydrochloride & 1 & 2 & (5) \\
\hline Mephenytoin & 2 & 1 & (6) \\
\hline Methylprednisolone & 1 & 2 & (5) \\
\hline Meticillin & 3 & 4 & (5) \\
\hline Metolazone & 3 & 4 & (6) \\
\hline Metyrapone & 1 & 2 & (6) \\
\hline Metyrosine & 4 & 3 & (6) \\
\hline Mibefradil dihydrochloride & 2 & 1 & (6) \\
\hline Milrinone & 3 & 4 & (5) \\
\hline Nystatin & 3 & 4 & (5) \\
\hline Omeprazole & 1 & 2 & $(5)$ \\
\hline Oxymetholone & 1 & 2 & (6) \\
\hline Oxytetracycline & 3 & 4 & (6) \\
\hline P-aminosalicylic acid & 1 & 2 & (5) \\
\hline Pancuronium bromide & 3 & 4 & (5) \\
\hline Penbutolol & 2 & 1 & (6) \\
\hline Phenylethylmalonamide & 3 & 4 & (5) \\
\hline Practolol & 3 & 4 & (6) \\
\hline Procainamide hydrochloride & 3 & 4 & $(5)$ \\
\hline Prochlorperazine & 1 & 2 & (5) \\
\hline
\end{tabular}


Table III. (continued)

\begin{tabular}{|c|c|c|c|}
\hline Name & Prior listed BDDCS & Corrected BDDCS & Initial publication \\
\hline Pyrimethamine & 3 & 1 & $(5)$ \\
\hline Quinapril hydrochloride & 2 & 1 & (5) \\
\hline Raltegravir potassium & 2 & 1 & (5) \\
\hline Regadenoson & 3 & 4 & (5) \\
\hline Repaglinide & 2 & 1 & (5) \\
\hline Ritodrine & 3 & 1 & (5) \\
\hline Roquinimex & 2 & 1 & (6) \\
\hline Sofosbuvir & 3 & 1 & (6) \\
\hline Sparfloxacin & 1 & 2 & (5) \\
\hline Talinolol & 3 & 4 & (5) \\
\hline Tedizolid phosphate & 1 & 2 & (6) \\
\hline Telithromycin & 2 & 1 & (5) \\
\hline Temafloxacin hydrochloride & 3 & 4 & (6) \\
\hline Temocapril hydrochloride & 1 & 2 & (5) \\
\hline Temocaprilat & 3 & 4 & (5) \\
\hline Temozolomide & 2 & 1 & (5) \\
\hline Temsirolimus & 1 & 2 & (5) \\
\hline Tenofovir disoproxil fumarate & 3 & 1 & (5) \\
\hline Tetrabenazine & 2 & 1 & (5) \\
\hline Thioridazine & 1 & 2 & (5) \\
\hline Tizanidine hydrochloride & 2 & 1 & (5) \\
\hline Triamcinolone acetonide & 1 & 2 & (5) \\
\hline Trovafloxacin mesylate & 1 & 3 & (6) \\
\hline Verapamil hydrochloride & 1 & 2 & (5) \\
\hline Zaleplon & 2 & 1 & (5) \\
\hline
\end{tabular}

unaffected by intestinal metabolism) will be close to 1.0; (d) BDDCS classes 1 and 2 (high permeability) base and neutral compounds (ECCS class 2) will be metabolized in rank order by CYP3A4>UGTs>CYP2D6>esterases,CYP2C; (e) base and neutral high permeability compounds (ECCS class 2) will be preferentially P-glycoprotein (P-gp) substrates affecting $F_{\text {abs }}$, the fraction of an oral dose that is absorbed; (f) low permeability acids and zwitterions with molecular weight < 400Da will be renally excreted (ECCS class 3A) while those acids and zwitterions with molecular weight $\geq 400$ Da will be rate-limited by OATP uptake, but eliminated predominantly by the renal route; $(\mathrm{g})$ low permeability bases and neutral compounds (ECCS class 4) will be excreted renally.

We concur that the ECCS system is a beneficial addition to BDDCS in predicting drug disposition and bioavailability, and that the addition of criteria related to substrate molecular weight and charge status is important. The addition of the many drugs for which BDDCS has been categorized as presented here, differentiating high from low permeability, should provide a fertile basis for further discoveries related to ECCS or other yet to be identified compound criteria. A major difference between BDDCS and ECCS is providing predictability based on solubility, which is not considered in ECCS, but is a critical determinant in BDDCS and BCS. We expand below on how the solubility criterion is important in predictions of drugs yielding central pharmacodynamics, drug-induced liver injury (DILI), and food effects.

\section{Improving the Prediction of the Brain Disposition for Drugs Using BDDCS}

Broccatelli et al. (14) identified 153 drugs that met three criteria: (a) the presence or absence of central human pharmacodynamic effects was known; (b) the drug's permeability/metabolism and BDDCS class had been assessed; and (c) experimental in vitro results were available as to whether the drug was or was not a substrate for P-gp (or ABCB1), since it is generally believed that P-gp substrates do not yield central effects (15). The authors found that 17 of the 153 drugs were high permeability BDDCS class 1 compounds that exhibited significant P-gp efflux in vitro. But all 17 of these P-gp substrates, including sertraline, verapamil, and zolmitriptan, exhibit central pharmacodynamic effects. This supports the conclusion for BDDCS class 1 drugs presented in Fig. $3 \mathrm{~B}$ that transporters are clinically insignificant, and that this also holds for other membranes, including the brain. To make such an assessment on the potential for blood-brain barrier permeability, the differentiation among high permeability compounds requires knowledge of a drug's solubility. The important implication of these results in drug development is that BDDCS class 1 compounds are likely to be brain permeable and achieve pharmacodynamically relevant concentrations, whether this is desired or not. This could be a strong rationale for not always wanting a class $1 \mathrm{NME}$. We have recently shown that almost all antidepressants (16) and antihypertensives (17) are BDDCS class 1 drugs.

\section{Using BDDCS to Validate the Usefulness of DILI Predictive Metrics}

DILI is the leading cause of drug failure in clinical trials and a major reason for drug withdrawals from the market. Idiosyncratic DILI is very complex: several mechanisms appear to induce an immune response, reactive metabolites appear to be involved in most idiosyncratic DILI, and DILI is dependent on both dose and extent of hepatic metabolism. 
Table IV The current BCS and BDDCS class for drugs where both assignments are available

\begin{tabular}{lll}
\hline Name & BCS & BDDCS \\
\hline
\end{tabular}

Abacavir sulfate

Acalabrutinib

Acetaminophen

Acetazolamide

Acetylsalicylic acid

Acyclovir sodium

Albendazole

Albuterol sulfate

Allopurinol

Alprenolol

Amantadine hydrochloride

Amiloride

Amiodarone hydrochloride

Amitriptyline hydrochloride

Amodiaquine

Amoxicillin

Amphotericin B

Antipyrine

Astemizole

Atenolol

Atorvastatin calcium

Atropine sulfate

Azathioprine

Azithromycin dihydrate

Baricitinib

Bendroflumethiazide

Benznidazole

Benzthiazide

Bidisomide

Biperiden

Buspirone hydrochloride

Caffeine

Captopril

Carbamazepine

Carvedilol

Cefazolin sodium

Cetirizine hydrochloride

Chloramphenicol

Chloroquine

Chlorothiazide sodium

Chlorpheniramine maleate

Chlorpromazine hydrochloride

Chlorthalidone

Chlorzoxazone

Cimetidine

Ciprofloxacin hydrochloride

Cisapride

Clofazimine

Clomiphene citrate

Clomipramine

Cloxacillin

Codeine monohydrate

Colchicine

Cyclophosphamide

Cyclosporine

Dacomitinib

Danazol

Dapsone

Darolutamide

Desipramine hydrochloride

$\begin{array}{ll}1 \mid 3 & 1 \\ 2 & 2 \\ 1 \mid 3 & 1 \\ 4 & 3 \\ 3 & 1 \\ 1 \mid 3 & 4 \\ 2 \mid 4 & 2\end{array}$

Table IV. (continued)

\begin{tabular}{|c|c|c|}
\hline Name & $\mathrm{BCS}$ & BDDCS \\
\hline Dexamethasone & $1 / 3$ & 1 \\
\hline Diazepam & 1 & 1 \\
\hline Diclofenac sodium & 2 & 2 \\
\hline Dicloxacillin & 3 & 3 \\
\hline Didanosine & 3 & 3 \\
\hline Diethylcarbamazine citrate & 1 & 0 \\
\hline Diflunisal & 2 & 2 \\
\hline Digoxin & $1 / 2$ & 3 \\
\hline Diloxanide furoate & $2 \mid 4$ & 2 \\
\hline Diltiazem & 1 & 1 \\
\hline Diphenhydramine hydrochloride & 1 & 1 \\
\hline Disopyramide & 1 & 3 \\
\hline Doravirine & 2 & 2 \\
\hline Doxepin hydrochloride & 1 & 1 \\
\hline Doxycycline hyclate & 1 & 3 \\
\hline Duvelisib & 4 & 2 \\
\hline Efavirenz & $2 \mid 4$ & 2 \\
\hline Elagolix sodium & 3 & 1 \\
\hline Enalapril & 1 & 1 \\
\hline Encorafenib & 2 & 2 \\
\hline Ephedrine & 1 & 3 \\
\hline Erdafitinib & 1 & 2 \\
\hline Ergonovine & $1 \mid 3$ & 1 \\
\hline Ergotamine tartrate & 3 & 2 \\
\hline Ertugliflozin & 1 & 1 \\
\hline Erythromycin & 213 & 3 \\
\hline Erythromycin lactobionate & 213 & 3 \\
\hline Erythromycin stearate & 213 & 4 \\
\hline Ethambutol hydrochloride & $1 \mid 3$ & 3 \\
\hline Ethinylestradiol & $1 / 3$ & 1 \\
\hline Ethosuximide & 1 & 1 \\
\hline Famotidine & 3 & 3 \\
\hline Fexofenadine hydrochloride & 3 & 3 \\
\hline Fluconazole & 1 & 3 \\
\hline Flufenamic acid & 2 & 2 \\
\hline Fluoxetine hydrochloride & 1 & 1 \\
\hline Flurbiprofen & 2 & 2 \\
\hline Folic acid & $2 \mid 4$ & 2 \\
\hline Fosamprenavir calcium & 1 & 2 \\
\hline Furosemide & $3 \mid 4$ & 4 \\
\hline Ganciclovir sodium & 3 & 3 \\
\hline Gilteritinib & 4 & 2 \\
\hline Glipizide & 2 & 2 \\
\hline Glucose & 1 & 1 \\
\hline Glyburide & 214 & 2 \\
\hline Griseofulvin & 2 & 2 \\
\hline Haloperidol & 214 & 2 \\
\hline Hydralazine hydrochloride & 3 & 1 \\
\hline Hydrochlorothiazide & $3 \mid 4$ & 3 \\
\hline Ibuprofen & 2 & 2 \\
\hline Imipramine hydrochloride & 1 & 1 \\
\hline Indinavir sulfate & 214 & 2 \\
\hline Indomethacin & 2 & 2 \\
\hline Iopanoic acid & 2 & 4 \\
\hline Isoniazid & 1 & 1 \\
\hline Isosorbide dinitrate & $1 / 3$ & 1 \\
\hline Itraconazole & 2 & 2 \\
\hline Ivermectin & 214 & 1 \\
\hline Ivosidenib & 2 & 2 \\
\hline Ketoconazole & 2 & 2 \\
\hline Ketoprofen & 1 & 2 \\
\hline
\end{tabular}


Table IV. (continued)

\begin{tabular}{|c|c|c|c|c|c|}
\hline Name & $\mathrm{BCS}$ & BDDCS & Name & $\mathrm{BCS}$ & BDDCS \\
\hline Ketorolac tromethamine & 1 & 3 & Penicillamine & 3 & 3 \\
\hline Labetalol & 1 & 1 & Penicillin V & 1 & 4 \\
\hline Lamivudine & $1 \mid 3$ & 3 & Phenazopyridine hydrochloride & 2 & 2 \\
\hline Lansoprazole & 2 & 2 & Phenobarbital & 1 & 1 \\
\hline Lemborexant & 2 & 2 & Phenylbutazone & 2 & 1 \\
\hline Letermovir & 2 & 4 & Phenytoin sodium & 2 & 2 \\
\hline Leucovorin calcium & 3 & 3 & Pindolol & 1 & 1 \\
\hline Levamisole & $1 / 3$ & 1 & Piroxicam & 2 & 2 \\
\hline Levodopa & 1 & 1 & Pravastatin sodium & 3 & 3 \\
\hline Levofloxacin & 1 & 3 & Praziquantel & 2 & 2 \\
\hline Levonorgestrel & 1 & 2 & Prednisolone & 1 & 1 \\
\hline Lidocaine & 1 & 1 & Primaquine & 1 & 1 \\
\hline Lisinopril & 3 & 3 & Probenecid & 2 & 2 \\
\hline Lithium carbonate & 1 & 3 & Prochlorperazine & 2 & 2 \\
\hline Lomefloxacin & 1 & 3 & Proguanil & 1 & 1 \\
\hline Loperamide hydrochloride & 4 & 1 & Promazine hydrochloride & 1 & 1 \\
\hline Lopinavir & $2 \mid 4$ & 2 & Promethazine hydrochloride & $1 / 3$ & 1 \\
\hline Lovastatin & 2 & 2 & Propranolol hydrochloride & 1 & 1 \\
\hline Macitentan & 2 & 2 & Propylthiouracil & 3 & 1 \\
\hline Maprotiline & 1 & 1 & Pyrantel pamoate & $2 \mid 4$ & 2 \\
\hline Mebendazole & $2 \mid 4$ & 2 & Pyrazinamide & 1 & 1 \\
\hline Meclizine hydrochloride & 4 & 1 & Pyridostigmine bromide & 3 & 3 \\
\hline Meclofenamic acid sodium & 2 & 2 & Pyrimethamine & 214 & 1 \\
\hline Mefenamic acid & 2 & 2 & Quinidine sulfate dihydrate & 1 & 1 \\
\hline Mefloquine & $2 \mid 4$ & 2 & Quinine bisulfate heptahydrate & $1 / 3$ & 1 \\
\hline Meperidine & 1 & 1 & Raloxifene & 2 & 2 \\
\hline Metformin hydrochloride & 3 & 3 & Ranitidine hydrochloride & 3 & 3 \\
\hline Methionine & 1 & 1 & Reserpine & 3 & 1 \\
\hline Methotrexate & $3 \mid 4$ & 3 & Ribociclib & 4 & 1 \\
\hline Methyldopa & 3 & 3 & Rifampin & 2 & 2 \\
\hline Metoclopramide hydrochloride & $1 / 3$ & 1 & Risperidone & 2 & 1 \\
\hline Metoprolol tartrate & 1 & 1 & Ritonavir & $2 \mid 4$ & 2 \\
\hline Metronidazole & 1 & 1 & Rosiglitazone maleate & 1 & 2 \\
\hline Miconazole nitrate & 4 & 2 & Salicylic acid & 1 & 1 \\
\hline Midazolam hydrochloride & 1 & 1 & Saquinavir methanesulfonate & 214 & 2 \\
\hline Minocycline hydrochloride & 1 & 1 & Sarecycline & 3 & 3 \\
\hline Misoprostol & 1 & 1 & Selinexor & 2 & 2 \\
\hline Morphine hydrochloride & $1 \mid 3$ & 1 & Selumetinib sulfate & 4 & 1 \\
\hline Nadolol & 3 & 3 & Semaglutide & 4 & 2 \\
\hline Nalidixic acid & 2 & 2 & Sertraline hydrochloride & 2 & 1 \\
\hline Naproxen sodium & 2 & 2 & Siponimod & 2 & 2 \\
\hline Nelfinavir & 214 & 2 & Sirolimus & 2 & 2 \\
\hline Neomycin b sulfate & 4 & 3 & Solriamfetol & 1 & 3 \\
\hline Neostigmine methylsulfate & 3 & 3 & Spironolactone & 214 & 2 \\
\hline Netupitant & 2 & 2 & Stavudine & 1 & 3 \\
\hline Nevirapine & 2 & 2 & Sulfadiazine & 214 & 4 \\
\hline Niacinamide & 1 & 1 & Sulfamethoxazole & 2 & 2 \\
\hline Niclosamide & $2 \mid 4$ & 4 & Sulfasalazine & 214 & 2 \\
\hline Nifedipine & $1 / 2$ & 2 & Sulindac & 2 & 2 \\
\hline Nifurtimox & 3 & 2 & Tacrolimus & 2 & 2 \\
\hline Nitrofurantoin & 2 & 4 & Talinolol & 2 & 4 \\
\hline Nitroglycerin & $1 \mid 3$ & 1 & Tamoxifen & 2 & 1 \\
\hline Norethindrone & 1 & 1 & Terfenadine & 214 & 2 \\
\hline Norfloxacin & 4 & 4 & Tetracycline hydrochloride & 3 & 3 \\
\hline Norgestrel & 1 & 1 & Theophylline anhydrous & 1 & 1 \\
\hline Nortriptyline & 1 & 1 & Thyroxine & 3 & 2 \\
\hline Nystatin & $3 \mid 4$ & 4 & Tolmetin & 2 & 2 \\
\hline Ofloxacin & 2 & 3 & Tramadol & 1 & 1 \\
\hline Orphenadrine & 1 & 1 & Trichlormethiazide & 3 & 3 \\
\hline Oxaprozin & 2 & 2 & Triclabendazole & 214 & 2 \\
\hline Papaverine hydrochloride & 2 & 1 & Trimethoprim & 213 & 3 \\
\hline
\end{tabular}

Table IV. (continued) 
Table IV. (continued)

\begin{tabular}{lll}
\hline Name & BCS & BDDCS \\
\hline Ubrogepant & 4 & 2 \\
Valproic acid & $1 / 2$ & 1 \\
Valsartan & 3 & 4 \\
Verapamil hydrochloride & $1 \mid 2$ & 2 \\
Vitamin A & $2 \mid 4$ & 2 \\
Vitamin B1 & 3 & 3 \\
Vitamin B2 & 1 & 4 \\
Vitamin B6 & 1 & 1 \\
Vitamin C & 3 & 0 \\
Vitamin D2 & 3 & 2 \\
Warfarin & $1 \mid 2$ & 2 \\
Zalcitabine & 3 & 3 \\
Zidovudine & 1 & 1 \\
\hline
\end{tabular}

Many toxicology efforts are dedicated to developing methodologies to predict DILI for an NME that are complex and time-consuming. However, we have found that these methodologies often do no better than just avoiding BDDCS class 2 compounds $(18,19)$. As seen in Fig. 4, with increasingly severe indicators of hepatic liability, more and more drugs fall into BDDCS class 2. In our analysis, none of the DILI predictive metrics, except keeping daily dose $<50 \mathrm{mg}$, provides any better prediction of DILI than just avoiding BDDCS class 2 drugs.

The advantage of the BDDCS system is that the BDDCS class prediction can be made before ever knowing the daily dose. However, many valuable BDDCS class 2 drugs do not cause DILI. Our papers $(18,19)$ explicitly state that BDDCS classification should not be used as a DILI predictive metric. But we emphasize that if a new DILI predictive metric cannot be differentiated from BDDCS class 2, there can be no confidence in the metric and the toxicity hypotheses implied. Toxicologists are not familiar with BDDCS or BCS and generally ignore our recommendations, spending considerable resources developing metrics that most often cannot be differentiated from this simple caution of avoiding BDDCS class 2 drugs. However recently, Brecklinghaus et al. (20), summarizing the collaborative effort of several academic and industry European and Mid-East toxicology units, recognized these observations writing: "In future, it will be important to study if readouts from in vitro tests e.g., cytotoxicity, carrier inhibition, gene expression alterations, reactive metabolite formation etc. will improve DILI prediction independent from BDDCS class. For this purpose, large sets of compounds (>100) with sufficient substances from all four BDDCS will be required."

\section{Predicting Food Effects Using BDDCS Prior to In Vivo Studies in Animals or Humans}

All approved drug products must be studied to determine the effects of high-fat meals on the bioavailability of the proposed dosage form, and this information is included in the drug label (21). In 1999, Fleisher et al. (22) summarized published studies examining the effects of high-fat meals on various BCS classified drugs as summarized in Fig. 5 adapted from Custodio et al. (7). Meals generally slow down stomach emptying causing the peak time $\left(T_{\text {peak }}\right)$ to increase with the highly soluble classes 1 and 3 compounds and most class 2 compounds. There were too few class 4 compounds to come to any conclusion. However, the extent of bioavailability $\left(F_{\text {extent }}\right)$ exhibited differences between class 1 drugs (where little change is observed), class 2 drugs where bioavailability is generally increased with a high-fat meal, and class 3 compounds where bioavailability is generally decreased. It is difficult to rationalize these findings as food effects and drug absorption are complicated processes. One might argue that high-fat meals would increase the intestinal concentrations of poorly soluble but highly permeable class 2 compounds and decrease the intestinal concentrations of highly soluble poorly permeable class 3 compounds, but why is no effect seen with highly soluble, highly permeable class 1 compounds? Custodio et al. (7) speculated that the outcomes were consistent with high-fat meals inhibiting intestinal efflux transporters, but we conclude that the outcome only appears to be predictive for about $70 \%$ of food effect studies. Recently, there has been interest in the ability of physiologically based pharmacokinetic (PBPK) modeling to predict food effects quantitatively, but the outcomes have not provided sufficient validation as reviewed in an FDAauthored publication (23). Most recently, Wagner et al. (24) examined the potential reasons for poor PBPK food effects predictions for two BDDCS class 2 drugs exhibiting increased $F_{\text {extent }}$ (pazopanib and ziprasidone) and a BDDCS class 3 drug exhibiting decreased $F_{\text {extent }}$ (trospium). Notice that these directional changes would have been correctly predicted following Fig. 5. The 2019 FDA-authored study (23) examined predictability for 39 drugs, but only 8 were identified. BDDCS and Fig. 5 would have predicted the direction of change correctly for 7 of the 8 (erring on nifedipine, a

Table V Changes in the classification of drugs when shifting from BCS to BDDCS: no change (yellow), moderate change (orange), complete change (red)

\begin{tabular}{c|c|c|c|c|}
\cline { 2 - 5 } \multicolumn{1}{c|}{} & BDDCS 1 & BDDCS 2 & BDDCS 3 & BDDCS 4 \\
\hline BCS 1 & 47 & 6 & 13 & 2 \\
BCS 2 & 5 & 52 & 2 & 5 \\
BCS 3 & 8 & 5 & 27 & 2 \\
BCS 4 & 5 & 6 & 2 & 4 \\
\hline
\end{tabular}


Table VI Drugs classified with the updated early solubility classification method

\begin{tabular}{lll}
\hline & Solubility $\mathbf{>} \mathbf{0 . 4 4} \mathbf{~ m g / m L}$ & Solubility $\leq \mathbf{0 . 4 4} \mathbf{~ m g / m L}$ \\
\hline BDDCS $\mathbf{0 1 1 | 3}$ & TRUE soluble $(637 ; 87.9 \%)$ & FALSE soluble $(88 ; 12.1 \%)$ \\
BDDCS $2 \mathbf{4}$ & FALSE insoluble $(37 ; 8.6 \%)$ & TRUE insoluble $(394 ; 91.4 \%)$ \\
\hline
\end{tabular}

BDDCS class 2 drug showing no significant change). We note that two of the drugs, ceritinib (designated BCS class 4) and cinnarizine (designated BCS class 2/4), are highly metabolized and BDDCS class 2 drugs with food effects causing increased $F_{\text {extent }}$ as per Fig. 5. We believe it is important to use BDDCS rather than BCS classification in evaluating these retrospective data due to the uncertainty of the in vitro permeability measures and the fact that BCS is based on permeability extent rather than permeability rate, where the latter is a better predictor of extent of metabolism. We still believe that there is not a sufficient number of BDDCS class 4 drugs studied to make any solid prediction, but our suggestion is increased $F_{\text {extent }}$. Predicting the presence of and the direction of food effects using BDDCS before an NME has been dosed to either animals or humans is a useful tool in preclinical drug development. BDDCS predictions are better than any animal food effect studies, and we recommend such animal studies should not be carried out. The field is a long way from predicting food effects quantitatively using PBPK approaches, and we recommend that regulatory agencies continue to require such studies in humans.

\section{A Major Routes of Drug Elimination

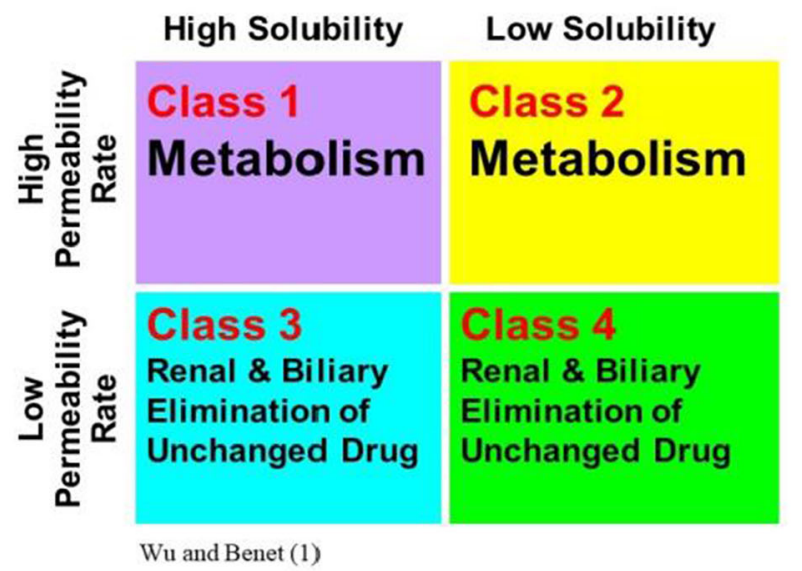

\section{B Prediction of Transporter Effects Based on BDDCS Class}

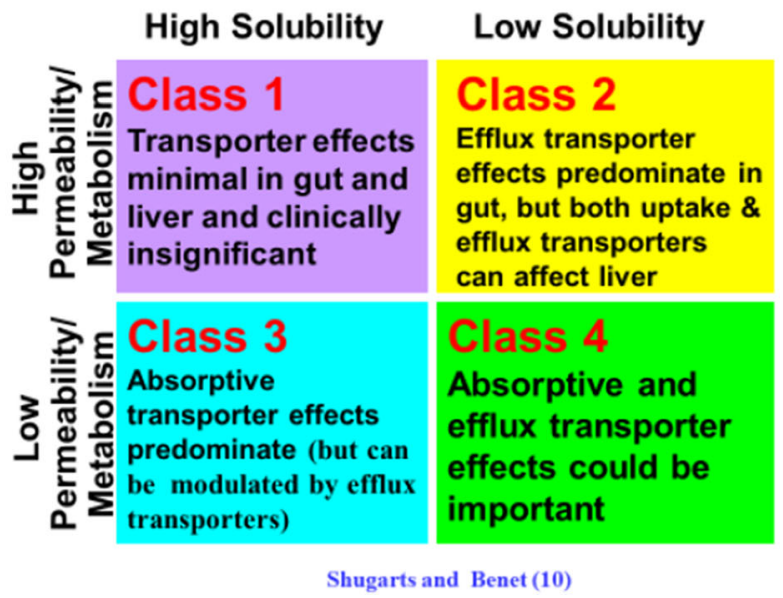

Fig. 3 Based on BDDCS: A Prediction of major route of drug elimination, B Prediction of transporter effects 


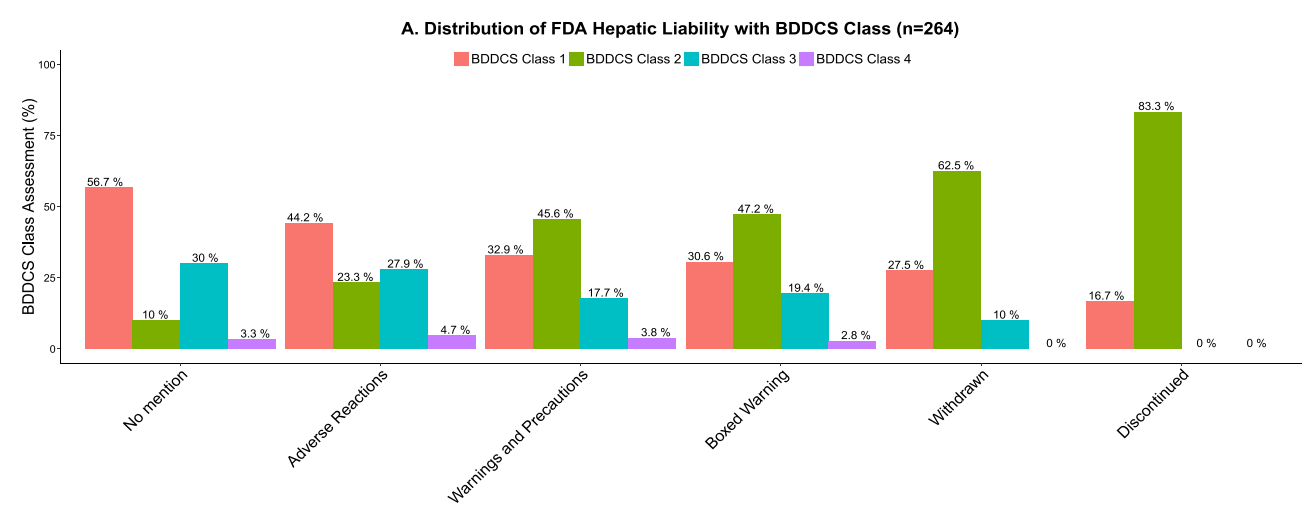

Fig. 4 Distribution by BDDCS class of hepatic liability for FDA listing of 264 drugs as reported by Chan and Benet (16)

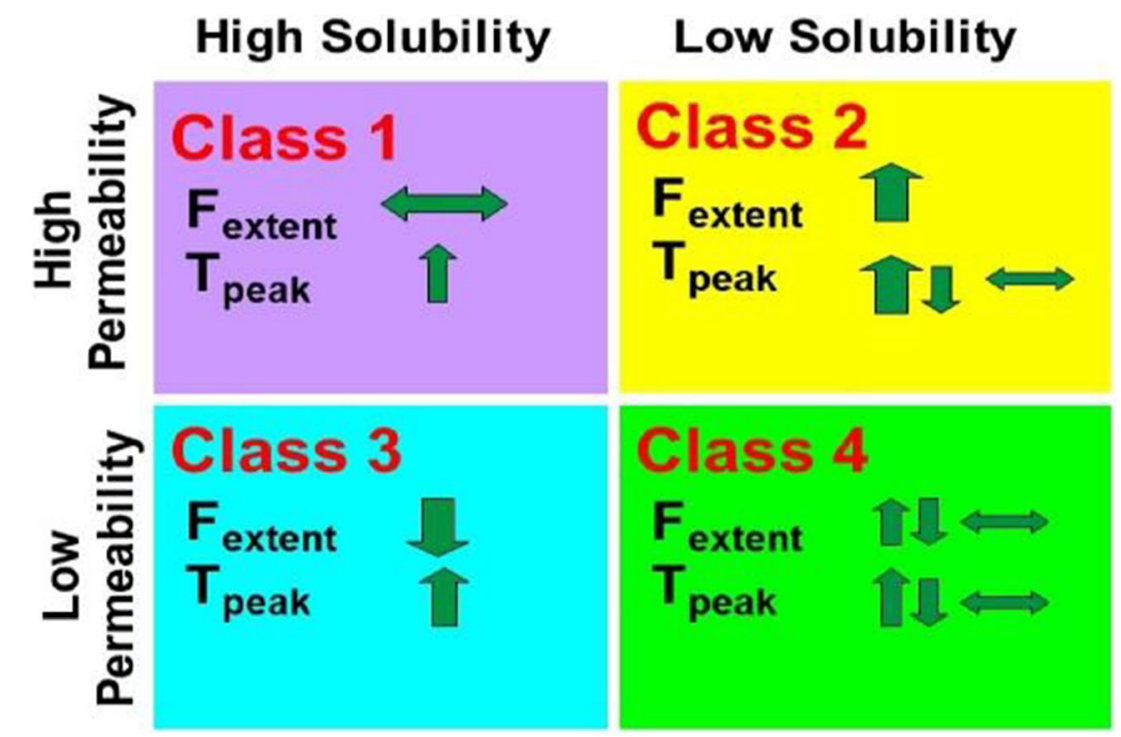

Fig. 5 Summary of the effects of high fat meals on the extent of bioavailability $\left(F_{\text {extent }}\right)$ and peak time $\left(T_{\text {peak }}\right)$ for BCS class drugs as presented by Fleisher et al. (20) adapted from Custodio et al. (7)

\section{RECOMMENDATION FOR BDDCS AND ECCS ASSIGNMENT EARLY IN DRUG DEVELOPMENT}

To differentiate BDDCS and ECCS classes 1 and 2 drugs from classes 3 and 4 drugs prior to dosing of an NME to animals and humans, it is necessary to have a reliable rate of permeability assay method that correctly differentiates a reasonably large set $(\geq 20)$ of approved drug formulations with known drug BDDCS and ECCS assignment. Then ECCS could be used to predict drug disposition class via molecular weight and charge. The almost 1500 drugs for which permeability is classified here can serve as the basis for further compound criteria discoveries beyond ECCS. With a $0.44-\mathrm{mg} / \mathrm{mL}$ water solubility cutoff, BDDCS assignments could inform further ECCS predictions, followed by additional predictions related to brain penetration, DILI potential, and food effects.

\section{CONCLUSIONS}

In this work, we have provided new BDDCS classification for 379 drugs, and we have described revisions for drugs that were already classified with BDCCS. We detail revised class assignment of previously misclassified drugs and references for the classification of new and previously classified drugs for maximum approved dose, extent of excretion of available drug excreted unchanged in the urine, and lowest solubility over the $\mathrm{pH}$ range $1.0-6.8$, when such information is available. We compare BDDCS and BCS classification for 257 BCS categorized drugs. We update the early development classification rule by increasing the solubility threshold from the original $0.3 \mathrm{mg} / \mathrm{mL}$ to the slightly more accurate $0.44 \mathrm{mg} /$ mL. We detail the uses of ECCS and BDDCS in predicting drug disposition characteristics prior to dosing animals or humans, the use of BDDCS to predict potential brain 
penetration, the outcome of food effect studies, and druginduced liver injury (DILI) potential. This work provides an update on the current status of the BDDCS and its uses in the drug development process.

\section{KEY TO UTILIZING THE SUPPLEMENTARY INFORMATION}

All data associated with this work is available in Supporting information Tables S1-4. Table S1 lists information for the BDDCS classified compounds: drug name, synonyms, CAS \#, year of approval, PubChem ID, SMILES, InChI, and charged state. Table S2 reports the current BDDCS assignment, and the parameters used to generate it, separated by collection: Benet et al. (5; as LZB2011), Hosey et al. (6; CMH2016), and the present additions (GB2021). In Table S3, the detailed revision of the data is reported. In the case of revisited drugs, both the former and the updated values are listed for fraction excreted unchanged in urine, maximum dose strength, solubility, dose number, and BDDCS assignment. For newly classified drugs, the new values only are reported in the columns labelled with [UPDATED]. If detected during the review process, the fraction of drug excreted unchanged in the bile is also reported. The drug transformation (i.e., whether the compound is a prodrug or an active metabolite) and the route of administration are also saved in this table. Additionally, in Table S3, metabolism and solubility data are assigned to a unique reference ID to provide an easy way to access the original data source. These IDs are listed in Table S4 along with the link to the original paper, drug label, etc. Table S5 lists the current BCS information for drugs. BCS classes were collected mainly from three publications: Lindenberg et al. (25), Wu and Benet (1), Box and Comer (26). When new BCS data were found from the inspection of FDA or EMA documents, we recorded and listed it as well (see GB2021 in Table S5). Finally, to facilitate the merging of the data across Tables S1-3 and 5, a unique ID (BDDCS.ID) is assigned to each compound in the collection and can be found in Tables $\mathrm{S} 1, \mathrm{~S} 2, \mathrm{~S} 3$, and S5.

\section{SUPPLEMENTARY INFORMATION}

The online version contains supplementary material available at https://doi.org/10.1208/s12248-022-00687-0.

\section{ACKNOWLEDGEMENTS}

The authors thank Benet Group members Alan R. Wolfe and Dr. Jasleen K. Sodhi for their critical review of the manuscript.

\section{AUTHOR CONTRIBUTION}

The initial evaluation and assignment of BDDCS classification was carried out by G.B., with review and revisions by L.Z.B and T.I.O. G.B. prepared the first draft, L.Z.B. the second draft, and further revisions by all authors.

\section{FUNDING}

This work was supported in part by the UCSF Benet Fund for Excellence generated from individual contributions and Dr. Benet's consultation, expert witness, and board of directors' fees that are made payable to the Regents of the University of California. Dr. Bocci work in the Benet Laboratory was funded by U24 CA224370. Dr. Oprea's participation was funded by U24 CA224370. Dr. Benet is a member of the UCSF Liver Center supported by NIH grant P30 DK026743.

\section{DECLARATIONS}

Competing Interests The authors declare no competing interests.

Open Access This article is licensed under a Creative Commons Attribution 4.0 International License, which permits use, sharing, adaptation, distribution and reproduction in any medium or format, as long as you give appropriate credit to the original author(s) and the source, provide a link to the Creative Commons licence, and indicate if changes were made. The images or other third party material in this article are included in the article's Creative Commons licence, unless indicated otherwise in a credit line to the material. If material is not included in the article's Creative Commons licence and your intended use is not permitted by statutory regulation or exceeds the permitted use, you will need to obtain permission directly from the copyright holder. To view a copy of this licence, visit http://creativecommons.org/licenses/by/4.0/.

\section{REFERENCES}

1. Wu C-Y, Benet LZ. Predicting drug disposition via application of BCS: transport/absorption/ elimination interplay and development of a biopharmaceutics drug disposition classification system. Pharm Res. 2005;22:11-23.

2. Amidon GL, Lennernäs H, Shah VP, Crison JR. A theoretical basis for a biopharmaceutic drug classification: the correlation of in vitro drug product dissolution and in vivo bioavailability. Pharm Res. 1995;12:413-20.

3. U.S. Department of Health and Human Services. Biopharmaceutics Classification System-Based Biowaivers Guidance for Industry [Internet]. In: Food and Drug Administration; 2021. Available from: https://www.fda.gov/media/ 148472/download.

4. Larregieu CA, Benet LZ. Drug discovery and regulatory considerations for improving in silico and in vitro predictions that use Caco-2 as a surrogate for human intestinal permeability measurements. AAPS J. 2013;15:483-97.

5. Benet LZ, Broccatelli F, Oprea TI. BDDCS applied to over 900 drugs. AAPS J. 2011;13:519-547.

6. Hosey CM, Chan R, Benet LZ. BDDCS predictions, selfcorrecting aspects of BDDCS assignments, BDDCS assignment corrections, and classification for more than 175 additional drugs. AAPS J. 2016;18:251-260.

7. Custodio JM, Wu C-Y, Benet LZ. Predicting drug disposition, absorption/elimination/transporter interplay and the role of food on drug absorption. Adv Drug Deliv Rev. 2008;60:717-33. 
8. Committee for Medicinal Products for Human Use. ICH M9 guideline on biopharmaceutics classification system-based biowaivers [Internet]. European Medicines Agency; 2020. Available from: https://www.ema.europa.eu/en/documents/scientific-guideline/ich-m9-biopharmaceutics-classification-systembased-biowaivers-step-5_en.pdf.

9. Metri M, Polli JE. Evaluation of excipient risk in BCS class I and III biowaivers. AAPS J. 2022;24:20.

10. Dave RA, Morris ME. Novel high/low solubility classification methods for new molecular entities. Int J Pharm. Elsevier. 2016;511:111-26.

11. Shugarts S, Benet LZ. The role of transporters in the pharmacokinetics of orally administered drugs. Pharm Res. 2009;26:2039-54.

12. Varma MV, Steyn SJ, Allerton C, El-Kattan AF. Predicting clearance mechanism in drug discovery: extended clearance classification system (ECCS). Pharm Res. 2015;32:3785-802.

13. Kimoto E, Mathialagan S, Tylaska L, Niosi M, Lin J, Carlo AA, Tess DA, Varma MVS. Organic Anion Transporter 2-mediated hepatic uptake contributes to the clearance of highpermeability-low-molecular-weight acid and zwitterion drugs: Evaluation using 25 drugs. J Pharmacol Exp Ther. 2018;367:322-34.

14. Broccatelli F, Larregieu CA, Cruciani G, Oprea TI, Benet LZ. Improving the prediction of the brain disposition for orally administered drugs using BDDCS. Adv Drug Deliv Rev. 2012;64:95-109.

15. Broccatelli F, Carosati E, Cruciani G, Oprea TI. Transportermediated efflux influences CNS side effects:ABCB1, from antitarget to target. Mol Inform. 2010;29:16-26.

16. Zheng Y, Chen X, Benet LZ. Reliability of in vitro and in vivo methods for predicting the effect of P-glycoprotein on the delivery of antidepressants to the brain. Clin Pharmacokinet. 2016;55:143-67.

17. Liu W, Yan T, Chen K, Yang L, Benet LZ, Zhai S. Predicting interactions between rifampin and antihypertensive drugs using the Biopharmaceutics Drug Disposition Classification System (BDDCS). Pharmacotherapy. 2020;40:274-90.

18. Chan R, Benet LZ. Evaluation of DILI predictive hypotheses in early drug development. Chem Res Toxicol. 2017;30:1017-29.
19. Chan R, Benet LZ. Evaluation of the relevance of DILI predictive hypotheses in early drug development: Review of in vitro methodologies vs. BDDCS classification. Toxicol Res. 2018;7:358-70.

20. Brecklinghaus T, Albrect W, Kappenberg F, Duda J, Vartak N, Edlund K, et al. The hepatocyte export carrier inhibition assay improves the separation of hepatotoxic from non-hepatotoxic compounds. Chem-Biol Interact. 2021; in press.

21. FDA. Assessing the effects of food on drugs in INDs and NDAs - clinical pharmacology considerations guidance for industry.2019. https://www.fda.gov/media/121313/download

22. Fleischer D, Li C, Zhou Y, Pao LH, Karim A. Drug, meal and formulation interactions influencing drug absorption after oral administration. Clinical implications. Clin Pharmacokinet. 1999;36:233-54.

23. Li M, Zhao P, Pan Y, Wagner C. Predictive performance of physiologically based pharmacokinetic models for the effect of food on oral drug absorption: current status. CPT Pharmacometrics Syst Pharmacol. 2018;7:82-9.

24. Wagner C, Kesisoglou F, Pepin XJH, Parrott P, Riedmaier AE. Use of physiologically based pharmacokinetic modeling for predicting drug-food interactions: Recommendations for improving predictive performance of low confidence food effect models. AAPS J. 2021;23:85.

25. Lindenberg M, Kopp S, Dressman JB. Classification of orally administered drugs on the World Health Organization Model list of Essential Medicines according to the biopharmaceutics classification system. Eur J Pharm Biopharm. 2004;58:265-278.

26. Box KJ, Comer JEA. Using measured $\mathrm{pKa}, \log \mathrm{P}$ and solubility to investigate supersaturation and predict BCS class. Curr Drug Metab. 2008;9:869-78.

Publisher's Note Springer Nature remains neutral with regard to jurisdictional claims in published maps and institutional affiliations. 